\title{
DISCRETE DIFFERENTIAL OPERATORS IN MULTIDIMENSIONAL HAAR WAVELET SPACES
}

\author{
CARLO CATTANI and LUIS M. SÁNCHEZ RUIZ
}

Received 30 July 2003

\begin{abstract}
We consider a class of discrete differential operators acting on multidimensional Haar wavelet basis with the aim of finding wavelet approximate solutions of partial differential problems. Although these operators depend on the interpolating method used for the Haar wavelets regularization and the scale dimension space, they can be easily used to define the space of approximate wavelet solutions.
\end{abstract}

2000 Mathematics Subject Classification: 35A35, 42C40, 41A15.

1. Introduction. Advantage has extensively taken upon wavelets in order to analyze a number of different applications, such as image processing, signal detection, geophysics, medicine, or turbulent flows $[8,9,10,13,14,15,18]$. More mathematically focussed differential equations and even nonlinear problems have also been studied with this cargo of possibilities that wavelets have brought out into scene $[1,2,6,16]$.

In this paper, we study a Cauchy problem with the PDE $u_{t}=L u$, where the unknown function $u(t, \mathbf{x}) \in L^{2}([0,1])$ for each $t \in[0, T], \mathbf{x} \in[0,1]^{d}, d \in \mathbb{N}$, and $L$ is a (nonnecessarily linear) partial differential operator whose derivatives act on the space variable. Situations like this happen quite commonly in evolution processes, such as fluid dynamics, heat transfer, elasticity, traffic flow, shock propagation, and so on [5, 12]. According to the nature and properties of $L$, different approaches have been developed, and even simple difference approximations may provide good results [3, 11]. However, in the presence of discontinuities some difficulties arise.

The development of Haar wavelets with its extreme suitability for dealing with experimental problems having piecewise constant functions as initial conditions or functions with sharp discontinuities, such as those that appear in the Riemann problem or within smooth environments, seems to provide a natural scheme to study these problems, and wavelet (approximate) solutions have been obtained by using some regular (mostly Daubechies) bases of wavelets [11].

In the following, we propose an algorithm based upon Haar wavelet basis in order to define a discrete operator that maps piecewise constant functions into piecewise constant functions, which projects the continuous operator $L$ therein, and enables to get wavelet approximate solutions in $L^{2}([0,1])$ just working with Haar wavelets.

2. Haar wavelet basis. Haar wavelets are piecewise constant functions with compact support and finite jumps at their extremal points. The drawback of not being smooth enough has been overcome by a smoothing process based on spline interpolation [3]. 
Haar functions $\varphi_{k}^{n}(x):=2^{n / 2} \varphi\left(2^{n} x-k\right)$ are generated from the scaling function $\varphi(x)$ which is given by the characteristic function $\chi_{[0,1]}$. Then the Haar family of wavelets $\psi_{k}^{n}(x):=\left\{2^{n / 2} \psi\left(2^{n} x-k\right)\right\}_{k, n \in \mathbb{Z}}$ fulfills

$$
\psi_{k}^{n}(x)= \begin{cases}2^{-n / 2}, & x \in\left[\frac{k}{2^{n}}, \frac{k+1 / 2}{2^{n}}\right), \\ -2^{-n / 2}, & x \in\left[\frac{k+1 / 2}{2^{n}}, \frac{k+1}{2^{n}}\right), \\ 0, & \text { elsewhere, }\end{cases}
$$

$\left\|\psi_{k}^{n}(x)\right\|_{2}=1$, and the multiresolution axioms [7], as well as the recursive conditions $2^{n+1 / 2} \varphi_{k}^{n}(x)=\varphi_{k}^{n+1}(x)+\varphi_{k+1}^{n+1}(x), \quad 2^{n+1 / 2} \psi_{k}^{n}(x)=\varphi_{k}^{n+1}(x)-\varphi_{k+1}^{n+1}(x), \quad k, n \in \mathbb{Z}$.

Haar wavelets form a complete orthonormal system for the (finite-energy) $L^{2}(\mathbb{R})$ functions [7] so that

$$
\begin{gathered}
L^{2}(\mathbb{R})=\bigoplus_{n \in \mathbb{Z}} W_{n}=V_{q} \oplus \bigoplus_{j \geq q} W_{j}, \quad q \in \mathbb{Z}, \\
V_{n+1}=V_{n} \oplus W_{n} .
\end{gathered}
$$

Here, for each fixed $n \in \mathbb{Z}, V_{n}$ is the subspace

$$
V_{n}:=\left\{f \in L^{2}(\mathbb{R}): f=\sum_{n, k \in \mathbb{Z}} c_{k}^{n} \chi_{D_{k}^{n}}, c_{k}^{n}=\text { constant }\right\},
$$

where $D_{k}^{n}=\left[k / 2^{n},(k+1) / 2^{n}\right)$ and $W_{n}$ are orthogonal subspaces in $L^{2}(\mathbb{R})$, the so-called wavelet spaces. As usual, we consider the scalar product

$$
\langle f, g\rangle:=\int_{-\infty}^{+\infty} f(x) \overline{g(x)} d x \quad\left(\forall f, g \in L^{2}(\mathbb{R})\right) .
$$

According to the first equation of (2.3), we have that $f(x)=\sum_{n, k \in \mathbb{Z}} \beta_{k}^{n} \psi_{k}^{n}(x)$. If we fix a resolution value $N=2^{M}, M \in \mathbb{N}$, in (2.3), then an approximation of the $L^{2}(\mathbb{R})$-space is obtained, for example, by $L^{2}(\mathbb{R}) \cong V_{0} \bigoplus_{n=0}^{N} W_{n}$, that is,

$$
f(x) \cong \pi^{N} f(x):=\alpha_{0}^{0}+\sum_{n=0}^{M-1} \sum_{k=0}^{2^{n}-1} \beta_{k}^{n} \psi_{k}^{n}(x),
$$

$\pi^{N}$ being a projection operator into $V_{N}$ so that $\pi^{N}: L^{2}(\mathbb{R}) \rightarrow V_{N}$. In general, the coefficients $\alpha_{n}^{k}, \beta_{k}^{n}$ are given by

$$
\alpha_{k}^{n}=\left\langle f, \varphi_{k}^{n}\right\rangle, \quad \beta_{k}^{n}=\left\langle f, \psi_{k}^{n}\right\rangle .
$$

The dyadic discretization is the operator $\nabla^{N}: L^{2}(\mathbb{R}) \rightarrow \mathbb{K}^{N} \subset \ell^{2}$, where $\mathbb{K}$ stands for the complex or real field, and $\nabla^{N} f(x)=\mathbf{f}^{N}=\left(f_{0}, f_{1}, \ldots, f_{N-1}\right)^{T} \in \mathbb{K}^{N}$ with $f_{k}:=$ $f(k /(N-1)), 0 \leq k \leq N-1$. 
The above notation may be simplified if we denote $\beta_{N}:=\left(\alpha_{0}^{0}, \beta_{0}^{0}, \beta_{0}^{1}, \ldots, \beta_{2^{M-1}-1}^{M-1}\right)$ and $\Psi_{N}(x):=\left(\varphi(x), \psi_{0}^{0}(x), \psi_{0}^{1}(x), \ldots, \psi_{2^{M-1}-1}^{M-1}(x)\right)$ so that

$$
\pi^{N} f(x)=\beta_{N} \Psi_{N}^{T}(x), \quad \mathbf{f}^{N}=\beta_{N} \nabla^{N} \Psi_{N}^{T}(x) .
$$

If $P_{2 i}$ denotes the permutation (or shuffle) matrix (cf. [3, 4, 7]) and $I$ is the identity matrix, then the discrete Haar wavelet transform is the one-to-one linear operator $\mathscr{H}$ of $\mathbb{K}^{N}$ onto $\mathbb{K}^{N}$ such that

$$
\mathscr{H} \mathbf{f}^{N}=\beta_{N}^{T}=\left[\prod_{k=1}^{M}\left(P_{2^{k}} \oplus I_{2^{M}-2^{k}}\right)\left(H_{2^{k}} \oplus I_{2^{M}-2^{k}}\right)\right] \mathbf{f}^{N} .
$$

The matrices $H_{2} i$ have size $2^{i} \times 2^{i}$ and are the direct sum of the lattice coefficients of the system (2.2),

$$
H_{2 i}:=\left(\begin{array}{cc}
2^{-1 / 2} & 2^{-1 / 2} \\
2^{-1 / 2} & -2^{-1 / 2}
\end{array}\right)_{1} \oplus \cdots \oplus\left(\begin{array}{cc}
2^{-1 / 2} & 2^{-1 / 2} \\
2^{-1 / 2} & -2^{-1 / 2}
\end{array}\right)_{2^{i}-1},
$$

where, for $1 \leq j \leq 2^{i}-1$ and $O_{p, q}$ standing for the $p \times q$ null matrix,

$$
\left(\begin{array}{cc}
2^{-1 / 2} & 2^{-1 / 2} \\
2^{-1 / 2} & -2^{-1 / 2}
\end{array}\right)_{j}=\left(\begin{array}{ccc}
O_{2 j-2,2 j-2} & O_{2 j-2,2} & O_{2 j-2,2^{i}-2 j} \\
O_{2,2 j-2} & \left(\begin{array}{cc}
2^{-1 / 2} & 2^{-1 / 2} \\
2^{-1 / 2} & -2^{-1 / 2}
\end{array}\right) & O_{2,2^{i}-2 j} \\
O_{2^{i}-2 j, 2 j-2} & O_{2^{i}-2 j, 2} & O_{2^{i}-2 j, 2^{i}-2 j}
\end{array}\right)
$$

Thus, having in mind that $V_{N+1} \simeq \mathbb{K}^{N}$ by identifying $\alpha_{0}^{0}+\sum_{n=0}^{M-1} \sum_{k=0}^{2^{n}-1} \beta_{k}^{n} \psi_{k}^{n}(x)$ with $\left\{\alpha_{0}^{0}, \beta_{k}^{n}\right\}_{k=0, \ldots, 2^{M-1}-1}^{n=0, \ldots, M-1}$, the projection operator $\pi^{N}: L^{2}(\mathbb{R}) \rightarrow V_{N+1}$ may be factorized by means of the following diagram:

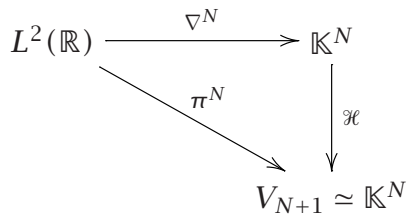

The operator $\pi^{N}$ maps each $f \in L^{2}(\mathbb{R})$ into a piecewise constant function $\pi^{N} f \in$ $V_{N+1}$, being easy to check $\lim _{N \rightarrow \infty} \pi^{N} f=f$ pointwise (cf. [7]).

Note that the inverse discrete Haar transform $\mathscr{H}^{-1}$ of (2.9) is univocally defined [7], but the inverse $\nabla^{-N}:=\left(\nabla^{N}\right)^{-1}$ of the operator $\nabla^{N}$ is not because there are infinitely many functions interpolating a finite set of values.

3. Haar series regularization. In what follows, we will consider the interpolating operator $\mathscr{S}^{p}: \mathbb{K}^{N} \rightarrow C^{p-1}(\Omega)$ which maps the vector $\mathbf{f}^{N}\left(=\left(f_{i}\right)_{i=0}^{N-1}\right)$, based on the dyadic nodes of $\Omega \subseteq \mathbb{R}$, into the $p$-differentiable function $s(x):=\mathscr{S}^{p} \mathbf{f}^{N}$, where $C^{-1}(\Omega)$ stands for the piecewise continuous functions defined on $\Omega$. Hence $s(x)$ is an interpolating function of order $p$ through $\left(i /(N-1), f_{i}\right), i=0, \ldots, N-1$. 
DEFINITION 3.1 (interpolating $p$-space). For a given resolution $N$, the interpolating $p$-space $\sigma^{p}$ is the space $\sigma^{p} \subset L^{2}(\Omega)$ of interpolating functions with (nonredundant) fixed conditions at the boundary

$$
\sigma^{p}:=\left\{s(x)=\mathscr{S}^{p} \mathbf{f}^{N} \text {, with } \mathscr{S}^{-p}:=\left(\mathscr{\varphi}^{p}\right)^{-1}=\nabla^{N}, \forall \mathbf{f}^{N} \in \mathbb{K}^{N}\right\} .
$$

There follows that, fixing the resolution $N$ and the interpolating method, each function in $\sigma^{p}$ corresponds one-to-one to a discrete set $\mathbf{f}^{N}$, making the following diagrams commutative:
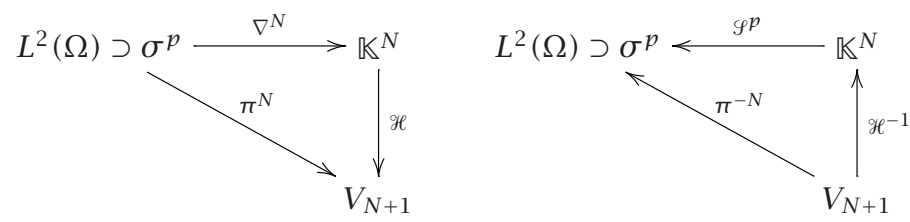

THEOREM 3.2. For a fixed resolution $N$ and a given interpolating method, the inverse $\pi^{-N}$ of the projection operator $\pi^{N}$ is univocally defined when acting on the $\sigma^{p}$ space functions by means of

$$
\pi^{-N}=\mathscr{S}^{p} \mathscr{H}^{-1} \quad\left(\pi^{N}=\mathscr{H}^{N}\right)
$$

Proof. Note that $\nabla^{N} \mathscr{\varphi}^{p}=\mathscr{\varphi}^{p} \nabla^{N}=I$ and $\mathscr{H}^{-1}$ is univocally defined [7].

DEFINITION 3.3. The $q$-derivative of a $p$-interpolating function, $0 \leq q \leq p$, at a given dimension $N$ is the linear operator $\delta^{(p, q)}: \mathbb{K}^{N} \rightarrow V_{N+1}$ such that

$$
\delta^{(p, q)}:=\pi^{N} \frac{d^{q}}{d x^{q}} \varphi^{p} .
$$

This definition provides an algorithm that maps piecewise constant functions into piecewise constant functions. If we look at it, we may note that firstly it smoothens piecewise constant functions with a suitable interpolation; secondly it finds out the derivative of the interpolating function, and then this derivative is transformed into a piecewise constant function by the discrete Haar wavelet transform.

From [7, page 13] and since $\left(d^{q} / d x^{q}\right) \mathscr{S}^{p} \nabla^{N} f$ belongs to $L^{2}(\Omega)$ for each $f \in L^{2}(\Omega)$, it follows that $\left\|\left(d^{q} / d x^{q}\right) \mathscr{S}^{p} \nabla^{N} f-\delta^{(p, q)} \nabla^{N} f\right\|_{L^{2}}^{2} \leq 2^{-2^{M-1}} 2^{\mu(\Omega)} K+\varepsilon$, where $\mu$ stands for the Lebesgue measure, $K=\sum_{k=0}^{M-1}\left(\left(m_{0, k, N}\right)^{2}+\left(m_{-1, k, N}\right)^{2}\right)^{1 / 2}$, with $m_{0, k, N}$ and $m_{-1, k, N}$ being the average value of $\left(d^{q} / d x^{q}\right) \mathscr{S}^{p} \nabla^{N} f$ over the first and the second half of $D_{k}^{N}$, respectively, and $\varepsilon$ may be taken as small as desired since any function in $L^{2}(\Omega)$ may be approximated by functions with compact support which are piecewise constant.

It is easy to see that if $f=\sum_{k=0}^{M-1} f_{k} X_{D_{k}}, g=\sum_{k=0}^{M-1} g_{k} X_{D_{k}} \in V_{N}$, where $f_{k}, g_{k}$ are constants, then there are unique $c_{k}$ such that $f_{k}=g_{k}+c_{k}$. Hence, there exists a matrix $A^{(p, q)}$ depending on $N$ that makes

$$
\begin{gathered}
\delta^{(p, q)} \nabla^{N} f=A^{(p, q)} \nabla^{N} f, \\
\delta^{(p, q)} \mathbf{f}^{N}=A^{(p, q)} \beta_{N} \nabla^{N} \Psi_{N}^{T}(x) .
\end{gathered}
$$


At the end of this paper, we provide an appendix with some of these matrices $A$ for different spline interpolations.

4. Multidimensional wavelet solution. In a $d$-dimensional variable space $\mathbf{x}=$ $\left(x_{1}, \ldots, x_{d}\right)$, we restrict ourselves to considering the same dyadic discretization on each coordinate of $\mathbf{x}$ so that the natural generalization of the dyadic discretization operator $\nabla^{N}: L^{2}\left(\mathbb{R}^{d}\right) \rightarrow \prod_{k=1}^{d} \mathbb{K}^{N}\left(N=2^{M}\right)$ is given by

$$
\nabla^{N} f(\mathbf{x}):=f\left(\frac{i_{1}}{N-1}, \frac{i_{2}}{N-1}, \ldots, \frac{i_{d}}{N-1}\right), \quad 0 \leq i_{j} \leq N-1,1 \leq j \leq d
$$

By using tensor products, we define $\mathbf{V}_{N}(\mathbf{x}):=\bigotimes_{i=1}^{d} V_{N}\left(x_{i}\right)$, where the (orthonormal) wavelet basis is $\left\{\psi_{k}^{n}\left(x_{1}\right) \otimes \cdots \otimes \psi_{k}^{n}\left(x_{d}\right)\right\}, 0 \leq k \leq N-1$ (cf. [17]). Thus $\mathbf{V}_{N}(\mathbf{x})$ is the space of the piecewise constant functions on the $d$-dimensional product $D_{k}^{N} \times D_{h}^{N}, 0 \leq h$, $k \leq 2^{N}-1$, which can be represented as

$$
\begin{aligned}
\pi^{N} f(\mathbf{x}):= & \prod_{1 \leq i \leq d} \alpha_{i} \varphi\left(x_{i}\right)+\sum_{\substack{0 \leq k_{i} \leq 2^{n_{i}} \\
0 \leq n_{i} \leq M \\
1 \leq i \leq d}} \prod_{1 \leq i \leq d} \beta_{k_{i}}^{n_{i}} \psi_{k_{i}}^{n_{i}}\left(x_{i}\right) \\
& +\sum_{\substack{0 \leq k_{i} \leq 2^{n_{i}-1} \\
0 \leq n_{i} \leq M \\
1 \leq i \leq d}} \prod_{\substack{1 \leq i, j \leq d \\
i \neq j}} \omega_{k_{i}, j}^{n_{i}} \varphi\left(x_{j}\right) \psi_{k_{i}}^{n_{i}}\left(x_{i}\right)
\end{aligned}
$$

in short, $\pi^{N} f(\mathbf{x}):=\sum_{0 \leq i_{j} \leq M-1,1 \leq j \leq d} \beta_{N}^{i_{1} i_{2} \cdots i_{d}} \nabla^{N}\left(\psi_{N}\left(x_{i_{1}}\right) \otimes \cdots \otimes \psi_{N}\left(x_{i_{d}}\right)\right)$.

Then the discrete $d$-dimensional Haar wavelet transform is the one-to-one linear operator $\mathscr{H}_{d}=\mathscr{H}_{\mathbb{2} \otimes} \stackrel{d}{ } \otimes \mathscr{H}$ of $\left(\mathbb{K}^{N}\right)^{d}$ onto $\left(\mathbb{K}^{N}\right)^{d}$, and the discrete derivative of $\nabla^{N} f(\mathbf{x})$ is the tensor product of the corresponding 1-dimensional discrete derivative. Hence, in the 2-dimensional case, this leads to a matrix $A^{(p, q)}$, which in fact is the same as the one of the 1-dimensional case, such that

$$
\begin{array}{ll}
\delta_{x}^{(p, q)} f\left(\frac{i_{1}}{N-1}, \frac{i_{2}}{N-1}\right)=A^{(p, q)} f\left(\cdot, \frac{i_{2}}{N-1}\right), & 0 \leq i_{2} \leq N-1, \\
\delta_{y}^{(p, q)} f\left(\frac{i_{1}}{N-1}, \frac{i_{2}}{N-1}\right)=A^{(p, q)}\left(\frac{i_{1}}{N-1}, \cdot\right), & 0 \leq i_{1} \leq N-1,
\end{array}
$$

proving that if $x_{1}=i_{1} /(N-1), x_{2}=i_{2} /(N-1)$,

$$
\delta_{x}^{(p, q)} \nabla^{N} f\left(x_{1}, x_{2}\right)=A^{(p, q)} \beta_{N} \nabla^{N} \Psi_{N}^{T}\left(x_{1}\right) \otimes \nabla^{N} \Psi_{N}^{T}\left(x_{2}\right),
$$

and analogously for $\delta_{y}^{(p, q)}$. Taking this into account, we obtain the following result. 
Proposition 4.1. An approximate wavelet solution $\tilde{u}(t, \mathbf{x})=\pi^{N} u(t, \mathbf{x})$ of the Cauchy problem

$$
u_{t}=L u, \quad u(0, \mathbf{x})=u_{0}(\mathbf{x}), \quad \mathbf{x} \in[0,1]^{d}, t \in[0, T]
$$

is obtained by taking

$$
\tilde{u}(t, \mathbf{x}):=\sum_{\substack{0 \leq i_{j} \leq M-1 \\ 1 \leq j \leq d}} \beta_{N}^{i_{1} i_{2} \cdots i_{d}}(t) \nabla^{N}\left(\psi_{N}\left(x_{i_{1}}\right) \otimes \cdots \otimes \psi_{N}\left(x_{i_{d}}\right)\right),
$$

where the wavelet coefficients are explicit functions of $t$.

Proof. From (4.5), it follows that $\pi_{N} u_{t}=\pi_{N} L u$. Hence, if $L_{\delta}$ denotes the operator acting on piecewise functions obtained by replacing the partial derivatives that appear on $L$ with the corresponding $\delta_{i_{j}}^{(p, q)} \nabla^{N}$, for example, if $L=L\left(\partial / \partial x, \partial^{2} / \partial x \partial y\right)$, then $L_{\delta}=L\left(\delta_{x}^{(p, 1)} \nabla^{N}, \delta_{y}^{(p, 1)} \delta_{x}^{(p, 1)} \nabla^{N}\right)$, it happens that the projection in $\mathbf{V}_{N}(\mathbf{x})$ must verify $\tilde{u}_{t}(t, \mathbf{x})=L_{\delta} \tilde{u}(t, \mathbf{x})$ and

$$
\sum_{\substack{0 \leq i_{j} \leq M-1 \\ 1 \leq j \leq d}} \beta_{N}^{i_{1} i_{2} \cdots i_{d}}(0) \nabla^{N}\left(\psi_{N}\left(x_{i_{1}}\right) \otimes \cdots \otimes \psi_{N}\left(x_{i_{d}}\right)\right)=\nabla^{N} f(\mathbf{x}) .
$$

Orthonormality of the scaling functions $\varphi$ and $\psi_{k}^{n}$ gives an easily solved first-order ordinary system in the unknown wavelet coefficients. Note that due to the definition of Haar wavelets, the boundary conditions are automatically fulfilled.

5. Spline Haar solution of a parabolic equation. In this section, we will compare the Haar regularized solution of the following 1-dimensional parabolic problem with the exact solution and the one obtained with Crank-Nicholson's method.

EXAMPLE 5.1. Compare at time 0.04 and 0.08 the above-mentioned methods in the heat equation, without exchanges at the boundary, and a periodic function as initial function given by

$$
\begin{gathered}
u_{t}=u_{x x}, \quad 0 \leqslant x \leqslant 1, t \geqq 0, \\
u(x, 0)=\sin (\pi x), \\
u(0, t)=u(1, t)=0 .
\end{gathered}
$$

In Figure 5.1, the exact solution $u(x, t)=\sin (\pi x) e^{-\pi^{2} t}$ at $t=0.01$ and the one obtained by the Haar regularized method based on 64 nodes are shown as functions of the variable distance, $x$. Table 5.1 compares the set of values that we obtain (HR) at time $t=0.04, t=0.08$ with the exact ones (E) and those obtained by Crank-Nicholson's method $(\mathrm{CN})$. 


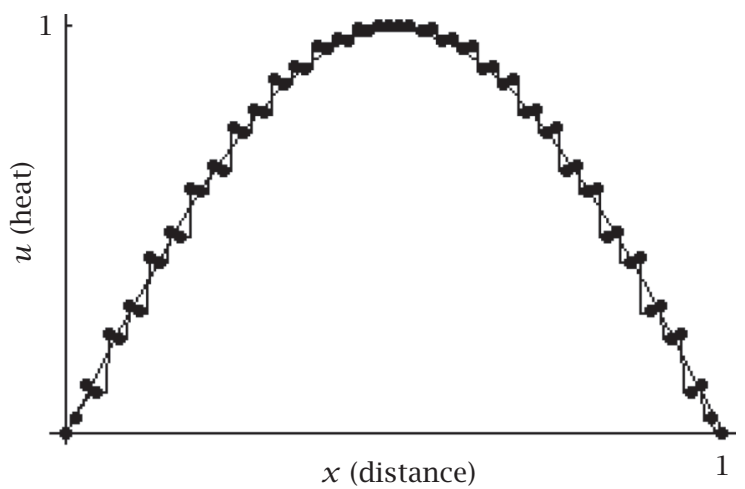

FIGURE 5.1. Exact and Haar regularized solution with 64 nodes.

TABLE 5.1

\begin{tabular}{l|cccccc}
\hline \multirow{2}{*}{$x$} & \multicolumn{3}{|c}{$t=0.04$} & \multicolumn{5}{c}{$t=0.08$} \\
\cline { 2 - 7 } & $\mathrm{E}$ & $\mathrm{HR}$ & $\mathrm{CN}$ & $\mathrm{E}$ & $\mathrm{HR}$ & $\mathrm{CN}$ \\
\hline 0 & 0 & 0 & 0 & 0 & 0 & 0 \\
0.2 & 0.396065 & 0.398 & 0.399 & 0.266878 & 0.269 & 0.271 \\
0.4 & 0.640846 & 0.648 & 0.646 & 0.431818 & 0.440 & 0.439 \\
0.6 & 0.640846 & 0.648 & 0.646 & 0.431818 & 0.440 & 0.439 \\
0.08 & 0.396065 & 0.398 & 0.399 & 0.266878 & 0.269 & 0.271 \\
1 & 0 & 0 & 0 & 0 & 0 & 0 \\
\hline
\end{tabular}

6. Annex. Finally, we enclose a list of matrices $A^{(p, q)}$, announced at the end of Section 3, which satisfy $\delta^{(p, q)} \nabla^{N} f=A^{(p, q)} \nabla^{N} f$. We fix our attention on the case $p=3$, $q=1$. For this and different resolutions $N=2^{M}, M \in \mathbb{N}$, the matrices $A^{(p, q)}$ are computed according to the following steps:

(1) discretize the interval $[0,1]$ at the dyadic points $k /(N-1), 0 \leq k \leq N-1$,

(2) consider the points whose first coordinates are the dyadic points and whose second coordinates are arbitrary values $a_{0}, a_{1}, \ldots, a_{N-1}$, that is, $\left(0, a_{0}\right),(1 /(N-$ $\left.1), a_{1}\right), \ldots,\left(1, a_{N-1}\right)$,

(3) build up the cubic spline that goes through the aforementioned set of points,

(4) compute the first derivative of this spline and evaluate it at the dyadic points,

(5) assuming the above evaluations generate the points $\left(0, a_{0}^{\prime}\right),\left(1 /(N-1), a_{1}^{\prime}\right), \ldots$, $\left(1, a_{N-1}^{\prime}\right)$, then $A^{(3,1)}$ is the $N \times N$ matrix that satisfies

$$
\left(\begin{array}{c}
a_{0}^{\prime} \\
a_{1}^{\prime} \\
\vdots \\
a_{N-1}^{\prime}
\end{array}\right)=A^{(3,1)}\left(\begin{array}{c}
a_{0} \\
a_{1} \\
\vdots \\
a_{N-1}
\end{array}\right)
$$


With a bound error of $10^{-5}$, routine calculations make $A^{(3,1)}$ become

$$
\begin{aligned}
& \left(\begin{array}{ll}
-0.16667 & 0.16667 \\
-0.16667 & 0.16667
\end{array}\right), \\
& N=2^{1}=2, \\
& \left(\begin{array}{cccc}
-0.97941 & 1.24412 & -0.34412 & 0.07941 \\
-2.14412 & 0.29118 & 2.40882 & -0.55588 \\
0.55588 & -2.40882 & -0.29118 & 2.14412 \\
-0.07941 & 0.34412 & -1.24412 & 0.97941
\end{array}\right), \\
& N=2^{2}=4, \\
& \left(\begin{array}{cccccccc}
-3.96693 & 5.02851 & -1.33162 & 0.31661 & -0.04656 & 0 & 0.00401 & -0.00401 \\
-4.56556 & 0.16762 & 5.51672 & -1.31167 & 0.19289 & 0 & -0.01662 & 0.01662 \\
1.22919 & -5.69897 & 0.26473 & 4.93006 & -0.72501 & 0 & 0.06246 & -0.06246 \\
-0.35120 & 1.62828 & -6.57564 & 2.59141 & 2.70715 & 0 & -0.23324 & 0.23324 \\
0 & 0 & 0 & 0 & -10.5 & 14 & -2.62951 & -0.87049 \\
0 & 0 & 0 & 0 & -3.5 & 0 & 0.25129 & 3.24871 \\
0 & 0 & 0 & 0 & 0 & 0 & -21 & 21 \\
0 & 0 & 0 & 0 & 0 & 0 & 0 & 0
\end{array}\right) \text {, } \\
& N=2^{3}=8 .
\end{aligned}
$$

ACKNOWLEDgment. The second author is supported by Generalitat Valenciana.

\section{REFERENCES}

[1] E. Bacry, S. Mallat, and G. Papanicolaou, A wavelet based space-time adaptive numerical method for partial differential equations, RAIRO Modél. Math. Anal. Numér. 26 (1992), no. 7, 793-834.

[2] W. Cai and J. Wang, Adaptive multiresolution collocation methods for initial-boundary value problems of nonlinear PDEs, SIAM J. Numer. Anal. 33 (1996), no. 3, 937-970.

[3] C. Cattani, Haar wavelet splines, J. Interdiscip. Math. 4 (2001), no. 1, 35-47.

[4] _ Haar wavelet-based technique for sharp jumps classification, Math. Comput. Modelling 39 (2004), no. 2-3, 255-278.

[5] R. Courant and K. O. Friedrichs, Supersonic Flow and Shock Waves, Interscience Publishers, New York, 1948.

[6] W. Dahmen, Wavelet methods for PDEs-some recent developments, J. Comput. Appl. Math. 128 (2001), no. 1-2, 133-185.

[7] I. Daubechies, Ten Lectures on Wavelets, CBMS-NSF Regional Conference Series in Applied Mathematics, vol. 61, Society for Industrial and Applied Mathematics, Pennsylvania, 1992.

[8] L. Debnath, Wavelet transforms and their applications, Proc. Indian Nat. Sci. Acad. Part A 64 (1998), no. 6, 685-713.

[9] R. A. DeVore, B. Jawerth, and B. J. Lucier, Image compression through wavelet transform coding, IEEE Trans. Inform. Theory 38 (1992), no. 2, 719-746.

[10] D. L. Donoho, Nonlinear wavelet methods for recovery of signals, densities, and spectra from indirect and noisy data, Different Perspectives on Wavelets (San Antonio, Tex, 
1993), Proc. Sympos. Appl. Math., vol. 47, American Mathematical Society, Rhode Island, 1993, pp. 173-205.

[11] S. Lazaar, P. Ponenti, J. Liandrat, and P. Tchamitchian, Wavelet algorithms for numerical resolution of partial differential equations, Comput. Methods Appl. Mech. Engrg. 116 (1994), no. 1-4, 309-314.

[12] R. J. LeVeque, Numerical Methods for Conservation Laws, 2nd ed., Lectures in Mathematics ETH Zürich, Birkhäuser, Basel, 1992.

[13] P. J. Oonincx, A wavelet method for detecting $S$-waves in seismic data, Comput. Geosci. 3 (1999), no. 2, 111-134.

[14] W. Schempp, Wavelet modelling of high resolution radar imaging and clinical magnetic resonance tomography, Results Math. 31 (1997), no. 3-4, 195-244.

[15] J. Segman and Y. Y. Zeevi, Image analysis by wavelet-type transforms: group-theoretic approach, J. Math. Imaging Vision 3 (1993), no. 1, 51-77.

[16] O. V. Vasilyev and S. Paolucci, A fast adaptive wavelet collocation algorithm for multidimensional PDEs, J. Comput. Phys. 138 (1997), no. 1, 16-56.

[17] P. Wojtaszczyk, A Mathematical Introduction to Wavelets, London Mathematical Society Student Texts, vol. 37, Cambridge University Press, Cambridge, 1997.

[18] V. Zimin and F. Hussain, Wavelet based model for small-scale turbulence, Phys. Fluids 7 (1995), no. 12, 2925-2927.

Carlo Cattani: Dipartimento di Scienze Farmaceutiche (DiFARMA), Università degli Studi di Salerno, Via Ponte Don Melillo, Invariante 11/C, 84084 Fisciano (Salerno), Italy

E-mail address: ccattani@unisa.it

Luis M. Sánchez Ruiz: Departamento de Matemática Aplicada, Escuela Técnica Superior de Ingeniería de Diseño (ETSID), Universidad Politécnica de Valencia, 46022 Valencia, Spain

E-mail address: 1msr@mat.upv.es 


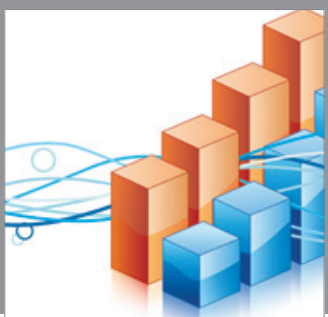

Advances in

Operations Research

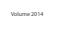

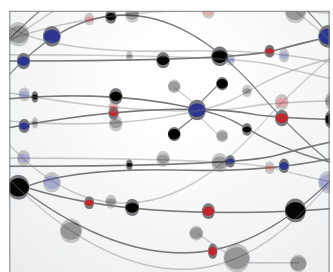

\section{The Scientific} World Journal
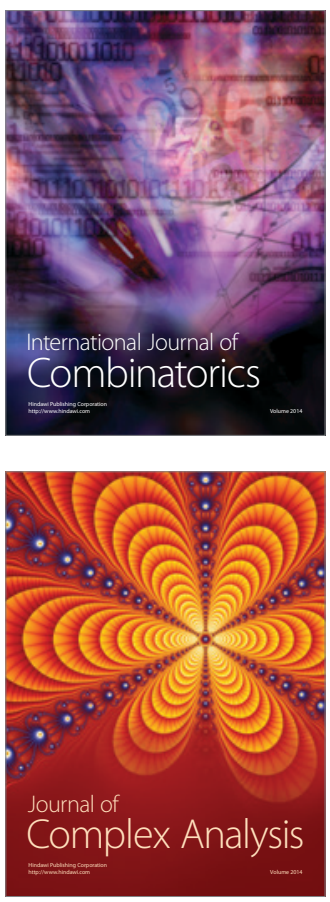

International Journal of

Mathematics and

Mathematical

Sciences
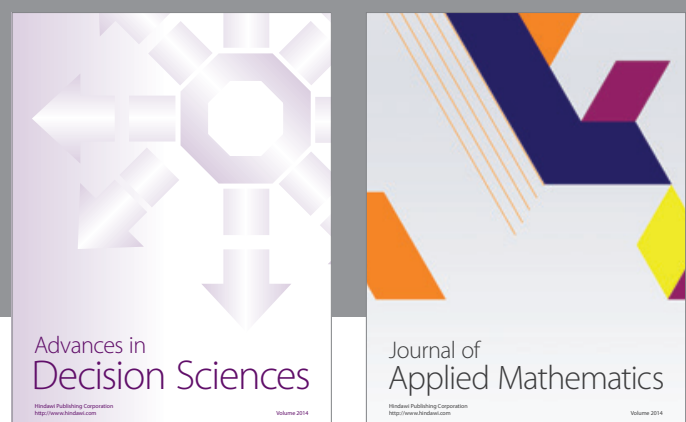

Journal of

Applied Mathematics
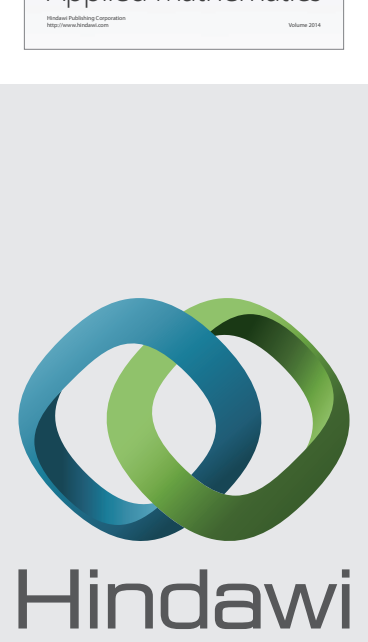

Submit your manuscripts at http://www.hindawi.com
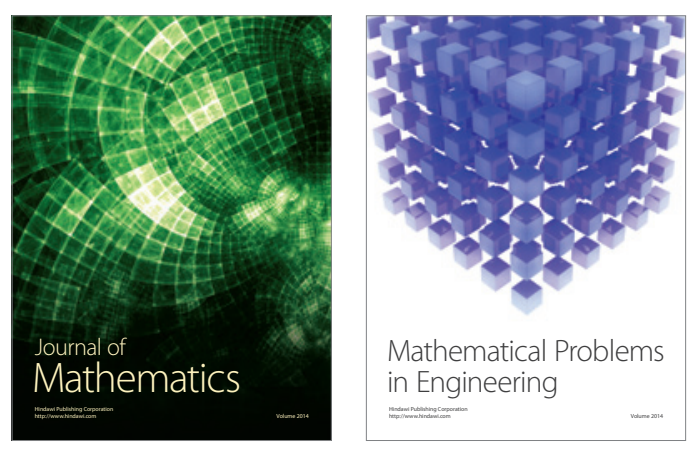

Mathematical Problems in Engineering
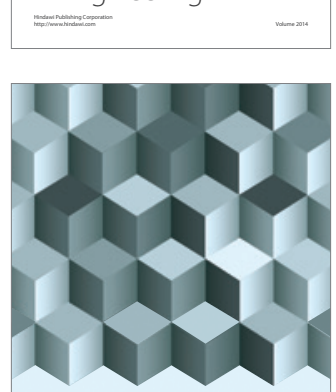

Journal of

Function Spaces
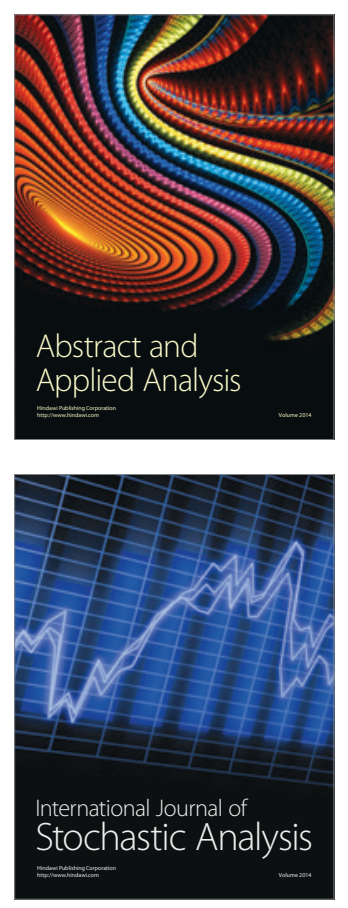

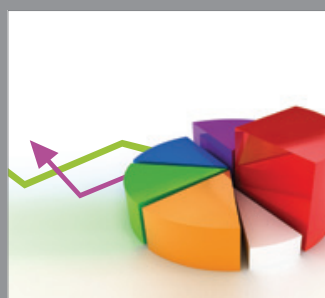

ournal of

Probability and Statistics

Promensencen
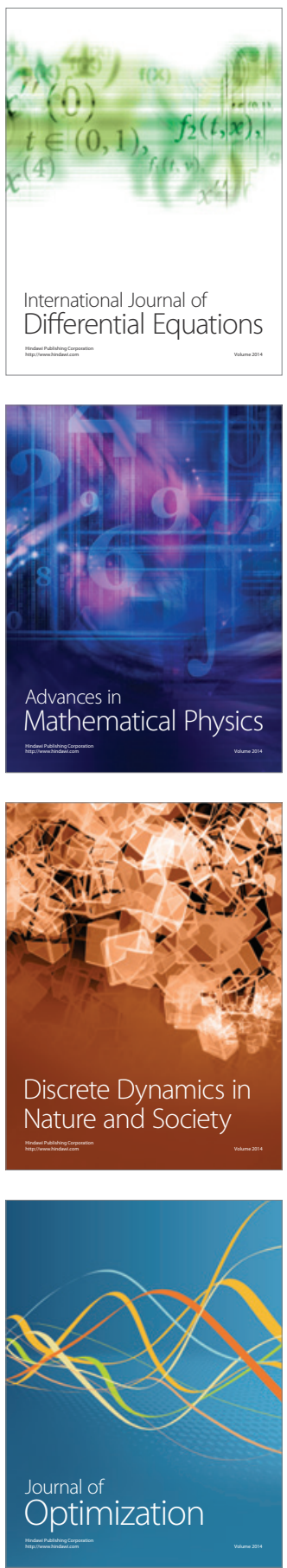